\title{
Article
}

\section{Weight loss interventions as an option for a lifestyle treatment in urinary incontinence}

Hill, James Edward, Christian, Danielle, Shaw, Kelly and Clegg, Andrew

Available at http://clok.uclan.ac.uk/35986/

Hill, James Edward ORCID: 0000-0003-1430-6927, Christian, Danielle ORCID: 0000-0003-1117-6127, Shaw, Kelly and Clegg, Andrew ORCID: 0000-00018938-7819 (2020) Weight loss interventions as an option for a lifestyle treatment in urinary incontinence. British Journal of Community Nursing, 25 (12). pp. 616-619. ISSN 1462-4753

It is advisable to refer to the publisher's version if you intend to cite from the work. 10.12968/bjen.2020.25.12.616

For more information about UCLan's research in this area go to http://www.uclan.ac.uk/researchgroups/ and search for <name of research Group>.

For information about Research generally at UCLan please go to http://www.uclan.ac.uk/research/

All outputs in CLoK are protected by Intellectual Property Rights law, including Copyright law. Copyright, IPR and Moral Rights for the works on this site are retained by the individual authors and/or other copyright owners. Terms and conditions for use of this material are defined in the policies page.

\section{CLoK}

Central Lancashire online Knowledge www.clok.uclan.ac.uk

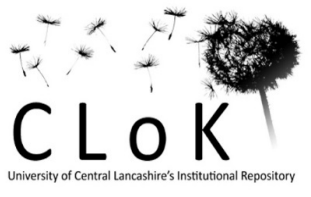




\section{Weight loss interventions may be considered as an option for a lifestyle treatment in urinary incontinence in adults.}

Keywords: lifestyle, urinary incontinence, weight loss, caffeine, fluid restrictions

\section{KEY POINTS}

- There were signs that weight loss management may be effective in improve symptoms in UI, specific urinary incontinence quality-of-life and prevalence of weekly UI.

- There is limited evidence on the effectiveness of caffeine reduction, or a soy-rich diet and no evidence found for physical activity, sweetened fizzy drinks or diet drinks, smoking cessation, constipation, and alcohol on urinary incontinence in adults

- Further high-quality random controlled trials are required to assess the effectiveness of lifestyle interventions for adults with UI.

- Future research for the effectiveness of lifestyle interventions for adults with urinary incontinence should ensure to use specific urinary incontinence outcome sets.

\section{Introduction}

Urinary incontinence (UI) (involuntary or abnormal urine loss) is a common condition affecting an estimated 423 million individuals around the world (Irwin et al. 2011). Its prevalence varies, ranging from between $5-15.2 \%$ in Asian, 1.8-30.5\% in European and 1.7-36.4\% in US populations (Milsom et al. 2014). This condition can have a significant clinical (Yang et al. 2018), psychological (Farage et al. 2008; Sims et al. 2011), and financial impact (Thom et al. 2010) on an individual's life. The risk of developing $\mathrm{UI}$ is associated with several non-modifiable modifiable risk factors of age (Milsom and Gyhagen 2019), gender (Nitti 2001) and family history (von Gontard et al. 2011) and modifiable risk 
factors of weight (Aune et al. 2019), smoking (Kawahara et al. 2020), diet (Maserejian et al. 2010) and caffeine intake (Gleason et al. 2013). Despite the association with such modifiable risk factors, there is limited evidence supporting interventions which aim to affect their influence (Imamura et al. 2015). This Cochrane systematic review aimed to evaluate the effectiveness of a range of lifestyle focused interventions which are commonly used in the management of UI.

Aims of commentary: This commentary critically appraises the methods used in the Cochrane systematic review and consider its importance of the findings for clinical practice.

\section{Methods}

This Cochrane systematic review undertook a comprehensive search of the Cochrane Central Register of Controlled Trials, and MEDLINE using a previously validated search procedure from date of inception to 3 July 2013. Additional handsearching, citation list and clinical trial registry searches were performed. There were no language restrictions placed on the search procedure. Only randomised control trials (RCTs) or quasi-randomised trials which compared lifestyle interventions to no active control treatment for adults with UI were included. A post hoc decision was made to include studies where all participants did not have to have UI on entering the trial.

A robust study selection, data extraction and assessment of risk of bias (Cochrane assessment of bias criteria) was undertaken independently by two reviewers with disagreements being resolved by discussion (Higgins 2020). A summary statement of quality for each outcome was produced using the GRADE criteria (Guyatt et al. 2008). A meta-analysis was undertaken using a fixed effect model, estimating relative risks for dichotomous outcomes and Mantel-Haenszel weighted mean differences for continuous outcomes. A range of subgroup analyses were planned but were not undertaken due to a lack of data. A narrative synthesis was undertaken for adverse events due to a similar lack of numerical data. 


\section{Findings}

Eleven studies were included of mixed design of which six were RCTs, four were crossover trials and one was a quasi-randomised trial. Out of the seven criteria for assessment of bias only two studies had a low risk of bias on four out of the seven criteria, namely randomisation, allocation concealment and blinding of outcome assessment and incomplete outcome data. The three main areas of concern were a lack of blinding of participants, appropriate allocation concealment and selective reporting bias due to limited protocols being identified for the included studies. Out of the 11 studies included, seven studies had mixed UI and non-diagnosed UI populations, ranging from $27 \%$ to $83 \%$ UI populations.

The review found that weight loss interventions resulted in adults with UI being statistically and clinically significantly more likely to report improvement in symptoms, incontinence impact questionnaire, cure rates by symptom quantification (low quality evidence: 1 studies) and improvement rates by symptom quantification compared to non-active controls (low quality evidence: 2 studies). On visual inspection of the forest plot for risk ratio of quantification of symptoms, there is some evidence that weight loss intervention becomes less effective over time (18 months follow-up), with the main effect moving towards the line of no effect. There was also a statistically and clinically significant reduction in prevalence of weekly UI (very low-quality evidence: 1 study). One study reported that the intervention had "few side effects".

One study demonstrated a statistical and clinically significant reduction in prevalence of weekly UI for lifestyle weight loss compared to the pharmacological intervention of metformin. For the intervention of restricting fluid intake there was mixed findings. There was one study out of three which demonstrated a statistically significant reduction in UI within a week (very low quality of evidence) and one study demonstrated improvement in quality of life compared to baseline measurements (very low quality of evidence). Two studies reported adverse events which included concentrated urine, constipation, headache, and thirst. Evidence on the effect's caffeine reduction and a soy-rich diet was limited and of low quality, preventing any conclusions. 


\section{Commentary}

Although the systematic review appeared robust when assessed using the Amstar2 critical appraisal tool (only considered inadequate on justification of study type selection), it had an important limitation that rendered it of "low confidence" (Shea et al. 2017). A post-hoc decision to include data from trials where not all participants were incontinent when they entered the trial, reduced the relevance of its findings to adults with UI. It is unclear as to when this decision was made, which may have led to important studies being missed during study selection. This limitation is further compounded by the limited and low quality evidence identified by the systematic review. Consequently, these findings should be viewed with caution.

Based on this limited evidence there are signs that weight loss interventions may be considered as an option for a lifestyle treatment due to some signs of effectiveness to improve symptoms in $\mathrm{UI}$, prevalence of weekly UI and there being limited adverse events. Previous systematic reviews have shown a positive association with increased BMI and risk of UI (Aune et al. 2019; Lamerton et al. 2018). Furthermore, previous reviews have shown that weight management interventions can be costeffective (Finkelstein and Verghese 2019; Forster et al. 2011; Loveman et al. 2011). However, the Cochrane systematic review found there was some evidence to suggest that the intervention of weight loss maybe less effective over time. This should be considered when recommending this intervention as regular assessments may be required to ensure continuation of effectiveness.

There was limited evidence to suggest a benefit from reducing fluid intake despite previous reviews finding the association between fluid intake and urinary frequency and urgency in men and women (Bradley et al. 2017). A range of adverse effects were also identified. As such reduced fluid intake interventions should be considered with caution.

There was limited or inconsistent evidence on the effectiveness of caffeine reduction or a soy-rich diet and no evidence found for physical activity, sweetened fizzy drinks or diet drinks, smoking cessation, 
constipation, and alcohol. Even though there are inconsistencies in the evidence base identified in this Cochrane review, the current NICE guidelines recommend that caffeine reduction, fluid modification and weight loss management should be considered (NICE 2015; 2019). Where these uncertainties still exist, there are other important aspects which can be taken into consideration such as sensitivity to the intervention, type of UI, patient preference and any caregiver needs (Demaagd and Davenport 2012; Lobchuk and Rosenberg 2014; Lukacz et al. 2017)

Further high-quality random controlled trials are required to assess the effectiveness of lifestyle interventions for adults with UI. Priority should be given to weight loss management due to this intervention type currently having the strongest evidence of effect. Due to the sparsity of the evidence, the areas of physical activity, sweetened fizzy drinks or diet drinks, smoking cessation, constipation, and alcohol should be explored. All future RCTs in this area should ensure that there is consistency in outcomes used by adopting relevant UI outcome sets for the type of UI (CHORUS 2020). Due to the scoping issues of this review and the out-of-date search procedure an update of this Cochrane review is required.

\section{CPD REFLECTIVE QUESTIONS}

- What was the main limitation of this Cochrane systematic review?

- What factors should be considered when prescribing lifestyle interventions for adults with urinary incontinence?

- What additional strategies can be put in place if uncertainties lie within the effectiveness of an intervention? 
This report is independent research funded by the National Institute for Health Research Applied Research Collaboration North West Coast (ARC NWC). The views expressed in this publication are those of the author(s) and not necessarily those of the National Institute for Health Research, the NHS, or the Department of Health and Social Care.

\section{References}

Aune D, Mahamat-Saleh Y, Norat T, Riboli E. 2019. Body mass index, abdominal fatness, weight gain and the risk of urinary incontinence: A systematic review and dose-response meta-analysis of prospective studies. BJOG : an international journal of obstetrics and gynaecology. 126(12):1424-1433.

Bradley CS, Erickson BA, Messersmith EE, Pelletier-Cameron A, Lai HH, Kreder KJ, Yang CC, Merion RM, Bavendam TG, Kirkali Z. 2017. Evidence of the impact of diet, fluid intake, caffeine, alcohol and tobacco on lower urinary tract symptoms: A systematic review. The Journal of urology. 198(5):1010-1020.

Developing a core outcome set for female pelvic floor disorders (urinary incontinence, pelvic organ prolapse, childbirth perineal trauma and pelvic pain syndromes). 2020. CHORUS; [accessed]. https://i-chorus.org/protocols-for-cos.

Demaagd GA, Davenport TC. 2012. Management of urinary incontinence. P \& T : a peer-reviewed journal for formulary management. 37(6):345-361h.

Farage MA, Miller KW, Berardesca E, Maibach HI. 2008. Psychosocial and societal burden of incontinence in the aged population: A review. Archives of gynecology and obstetrics. 277(4):285-290.

Finkelstein EA, Verghese NR. 2019. Incremental cost-effectiveness of evidence-based non-surgical weight loss strategies. Clinical obesity. 9(2):e12294.

Forster M, Veerman JL, Barendregt JJ, Vos T. 2011. Cost-effectiveness of diet and exercise interventions to reduce overweight and obesity. International journal of obesity (2005). 35(8):1071-1078.

Gleason JL, Richter HE, Redden DT, Goode PS, Burgio KL, Markland AD. 2013. Caffeine and urinary incontinence in us women. International urogynecology journal. 24(2):295-302.

Guyatt GH, Oxman AD, Kunz R, Vist GE, Falck-Ytter Y, Schünemann HJ. 2008. What is "quality of evidence" and why is it important to clinicians? BMJ. 336(7651):995.

Higgins JT, J.; Chandler, J.; Cumpston, M.; Li,T.; Page, MJ.; Welch, VA.; . 2020. Cochrane handbook for systematic reviews of interventions version 6.1. Cochrane.

Imamura M, Williams K, Wells M, McGrother C. 2015. Lifestyle interventions for the treatment of urinary incontinence in adults. Cochrane Database Syst Rev. (12):CD003505.

Irwin DE, Kopp ZS, Agatep B, Milsom I, Abrams P. 2011. Worldwide prevalence estimates of lower urinary tract symptoms, overactive bladder, urinary incontinence and bladder outlet obstruction. BJU Int. 108(7):1132-1138.

Kawahara T, Ito H, Yao M, Uemura H. 2020. Impact of smoking habit on overactive bladder symptoms and incontinence in women. Int J Urol. 
Lamerton TJ, Torquati L, Brown WJ. 2018. Overweight and obesity as major, modifiable risk factors for urinary incontinence in young to mid-aged women: A systematic review and meta-analysis. Obesity reviews : an official journal of the International Association for the Study of Obesity. 19(12):1735-1745.

Lobchuk MM, Rosenberg F. 2014. A qualitative analysis of individual and family caregiver responses to the impact of urinary incontinence on quality of life. Journal of wound, ostomy, and continence nursing : official publication of The Wound, Ostomy and Continence Nurses Society. 41(6):589-596.

Loveman E, Frampton GK, Shepherd J, Picot J, Cooper K, Bryant J, Welch K, Clegg A. 2011. The clinical effectiveness and cost-effectiveness of long-term weight management schemes for adults: A systematic review. Health technology assesIsment (Winchester, England). 15(2):1-182.

Lukacz ES, Santiago-Lastra Y, Albo ME, Brubaker L. 2017. Urinary incontinence in women: A review. JAMA. 318(16):1592-1604.

Maserejian NN, Giovannucci EL, McVary KT, McGrother C, McKinlay JB. 2010. Dietary macronutrient and energy intake and urinary incontinence in women. American journal of epidemiology. 171(10):1116-1125.

Milsom I, Coyne KS, Nicholson S, Kvasz M, Chen Cl, Wein AJ. 2014. Global prevalence and economic burden of urgency urinary incontinence: A systematic review. Eur Urol. 65(1):79-95.

Milsom I, Gyhagen M. 2019. The prevalence of urinary incontinence. Climacteric. 22(3):217-222.

Urinary incontinence in women quality standard [qs77]. 2015. NICE; [accessed]. https://www.nice.org.uk/guidance/qs77.

Urinary incontinence and pelvic organ prolapse in women: Management : Nice guideline [ng123]. 2019. [accessed]. https://www.nice.org.uk/guidance/ng123.

Nitti VW. 2001. The prevalence of urinary incontinence. Rev Urol. 3 Suppl 1(Suppl 1):S2-S6.

Sims J, Browning C, Lundgren-Lindquist B, Kendig H. 2011. Urinary incontinence in a community sample of older adults: Prevalence and impact on quality of life. Disabil Rehabil. 33(1516):1389-1398.

Thom DH, Brown JS, Schembri M, Ragins Al, Subak LL, Van Den Eeden SK. 2010. Incidence of and risk factors for change in urinary incontinence status in a prospective cohort of middle-aged and older women: The reproductive risk of incontinence study in kaiser. The Journal of urology. 184(4):1394-1401.

von Gontard A, Heron J, Joinson C. 2011. Family history of nocturnal enuresis and urinary incontinence: Results from a large epidemiological study. The Journal of urology. 185(6):23032306.

Yang E, Lisha NE, Walter L, Obedin-Maliver J, Huang AJ. 2018. Urinary incontinence in a national cohort of older women: Implications for caregiving and care dependence. J Womens Health (Larchmt). 27(9):1097-1103. 